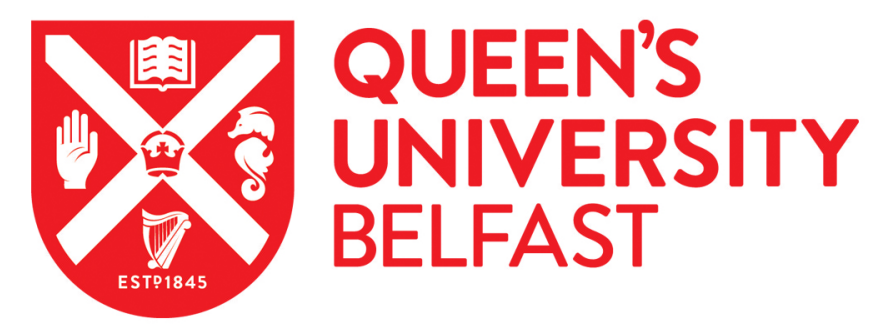

\title{
Two-Stage Hybrid Precoding in FDD Massive MIMO Systems with Low Rank Correlated Rician Fading Channels
}

\author{
Almradi, A., Matthaiou, M., Xiao, P., \& Fusco, V. (2020). Two-Stage Hybrid Precoding in FDD Massive MIMO \\ Systems with Low Rank Correlated Rician Fading Channels. In IEEE International Conference on \\ Communications: Proceedings (IEEE International Conference on Communications Workshops (ICC \\ Workshops): Proceedings). Institute of Electrical and Electronics Engineers Inc.. \\ https://doi.org/10.1109/ICCWorkshops49005.2020.9145278 \\ Published in: \\ IEEE International Conference on Communications: Proceedings
}

Document Version:

Peer reviewed version

Queen's University Belfast - Research Portal:

Link to publication record in Queen's University Belfast Research Portal

Publisher rights
(C) 2020 IEEE.
This work is made available online in accordance with the publisher's policies. Please refer to any applicable terms of use of the publisher.

General rights

Copyright for the publications made accessible via the Queen's University Belfast Research Portal is retained by the author(s) and / or other copyright owners and it is a condition of accessing these publications that users recognise and abide by the legal requirements associated with these rights.

Take down policy

The Research Portal is Queen's institutional repository that provides access to Queen's research output. Every effort has been made to ensure that content in the Research Portal does not infringe any person's rights, or applicable UK laws. If you discover content in the Research Portal that you believe breaches copyright or violates any law, please contact openaccess@qub.ac.uk. 


\title{
Two-Stage Hybrid Precoding in FDD Massive MIMO Systems with Low Rank Correlated Rician Fading Channels
}

\author{
Ahmed Almradi ${ }^{\dagger}$, Michail Matthaiou ${ }^{\dagger}$, Pei Xiao ${ }^{\ddagger}$, and Vincent F. Fusco ${ }^{\dagger}$ \\ ${ }^{\dagger}$ Institute of Electronics, Communications and Information Technology (ECIT), Queen's University Belfast, U.K. \\ $\ddagger$ The 5G Innovation Centre, Institute for Communication Systems, University of Surrey, U.K. \\ Emails: \{a.almradi, m.matthaiou\}@qub.ac.uk, p.xiao@surrey.ac.uk, and v.fusco@ecit.qub.ac.uk.
}

\begin{abstract}
To alleviate the downlink training and uplink feedback overhead in frequency-division duplexing massive multiple-input multiple-output systems, a two-stage precoder is proposed for hybrid analog and digital precoding. The analog beamformer and digital precoder are jointly designed by leveraging the signal-to-leakage-plus-noise ratio metric. The design of the analog beamformer is based only on the longterm channel statistics information, exploiting both the channel mean and low rank covariance statistics, whereas the digital precoder utilizes the knowledge of an effective channel with significantly reduced dimensionality. Consequently, we can considerably reduce the signalling and feedback overhead to the dimensions of the resultant effective channel. For comparison purposes, the cases of full channel state information at the transmitter (CSIT) and statistical CSIT are also investigated. It is shown that the digital precoder design problem reduces to the generalized Rayleigh quotient problem, while the analog beamformer design problem reduces to the quotient trace problem. These dimensionality reduction problems are solved via the generalized eigenvalue decomposition method.
\end{abstract}

\section{INTRODUCTION}

M ULTIUSER massive multiple-input multiple-output (MIMO) systems have witnessed an upsurge of research interest due to their ability to significantly improve the cellular spectral efficiency [1]-[3]. However, the high cost and power consumption of radio-frequency (RF) chains makes the fully digital structure costly. To alleviate this fundamental RF hardware constraint, hybrid precoding transceivers have been recently investigated, where a small number of RF chains are connected to a massive number of antennas through a set of low power and cost analog phase shifters. These two-stage structures divide the precoding and beamforming processing between the digital baseband and analog RF domains, respectively [1]. The huge potential of massive MIMO relies heavily on accurate channel state information at the transmitter (CSIT). In order to have full CSIT in frequency-division duplexing (FDD) systems, the channel has to be estimated at each user terminal and then fed back to the base station (BS) via a low-rate feedback channel. The bottleneck with FDD systems is that the training and feedback overhead resources scale with

This work was supported in part by the U.K. Engineering and Physical Sciences Research Council (EPSRC) under Grant EP/P000673/1 and Grant EP/N020391/1. the number of BS antennas, resulting in prohibitive CSIT acquisition overhead in massive MIMO systems [4]-[6]. Therefore, to reap the benefits of massive MIMO in FDD systems, channel dimensionality reduction techniques have been proposed. In this context, a two-stage technique called joint spatial division and multiplexing (JSDM) was proposed in [5]. In this work, channel dimensionality reduction was performed in two-stages; a pre-beamforming stage, that relies only on the channel second-order statistics to separate the users into groups according to their correlation structure similarities, by exploiting the block diagonalization technique, cascaded with a multiuser MIMO precoding stage which eliminates multiuser interference within each group, designed based on full CSIT of the reduced dimensionality channel.

The hybrid structure makes it infeasible to acquire the entire channel matrix of all antennas, since the channel estimator in the baseband pinpoints only to a reduced dimensionality channel through fewer number of RF chains. The two-stage CSIT structure, where the pre-beamforming stage is implemented in the analog RF domain and the multiuser MIMO precoding stage is realized in the digital baseband stage, was investigated in [2]. In this work, the analog beamformer is designed based only on the channel covariance matrices, by utilizing the averaged signal-toleakage-plus-noise ratio (SLNR) metric, whereas the digital precoder is obtained by employing the regularized zeroforcing (ZF) technique, exploiting only full CSIT of the reduced dimensionality effective channel. In addition, the authors in [7] used the long-term channel covariance matrices for the design of the digital precoder, while the analog beamformer is designed by beam search based on a beamsteering codebook. However, in the presence of strong line-of-sight (LOS) components, a predominant feature in future cellular small cells, dense networks and millimeter wave frequencies, a correlated Rician fading channel model needs to be considered. In this context, the authors in [8], [9] exploited the knowledge of channel mean information at the transmitter to introduce a sub-optimal precoder for the traditional fully digital structure. Therefore, motivated by the fact that the angular spread of the incoming and/or outgoing multipaths at the BS is small, massive MIMO 
channels often tend to have low rank structure. Hence, here we consider a two-stage hybrid precoding with rank deficient correlated Rician fading channels. Consequently, in the two-stage precoder, the analog beamformer is determined by the knowledge of the direct paths (LOS) components and low rank covariance matrices of the users, whereas the digital precoder utilizes perfect CSIT of the reduced dimensionality effective channel. Thus, the issue of user grouping, where all users in each group have the same covariance matrices (see e.g., [5], [10]), ceases to exist. Notice that in order to schedule a group of users which share similar covariance space, a massive number of users is indispensable.

\section{System AND Channel Model}

Consider a single-cell multiuser MIMO downlink system, where a BS equipped with $N_{B S}$ antennas and $N_{R F}$ RF chains serves $U$ single antenna users, where $U \leq N_{R F} \leq$ $N_{B S}$. A hybrid analog RF beamformer $\boldsymbol{F} \in \mathbb{C}^{N_{B S} \times N_{R F}}$ and a digital baseband precoder $\boldsymbol{W} \in \mathbb{C}^{N_{R F} \times U}$ are deployed at the BS. As a network of phase shifters is used to represent the analog RF beamforming stage, its entries are restricted to have a constant modulus, i.e., $\left|[\boldsymbol{F}]_{m, n}\right|=\frac{1}{\sqrt{N_{B S}}}$, where $|\cdot|$ denote the absolute value operator.

To explicitly take into account the dominant LOS components in addition to the low rank channel correlation, the $k^{\text {th }}$ user downlink channel model is defined as [11]

$$
\boldsymbol{h}_{k}=\sqrt{\frac{K_{k}}{K_{k}+1}} \boldsymbol{h}_{\mathrm{LOS}, k}+\sqrt{\frac{1}{K_{k}+1}} \boldsymbol{U}_{k} \boldsymbol{\Lambda}_{k}^{\frac{1}{2}} \boldsymbol{h}_{\mathrm{NLOS}, k},
$$

where $K_{k}$ is the ratio between the specular LOS and scattered non-LOS channel components, known as the Rician $\mathrm{K}$-factor for the $k^{\text {th }}$ user, $\boldsymbol{\Sigma}_{k}=\boldsymbol{U}_{k} \boldsymbol{\Lambda}_{k} \boldsymbol{U}_{k}^{\dagger}$ is the eigenvalue decomposition for the $k^{\text {th }}$ user channel covariance matrix, $\boldsymbol{\Lambda}_{k}$ is an $r_{k} \times r_{k}$ diagonal matrix whose entries are the $r_{k}$ nonzero dominant eigenvalues of $\boldsymbol{\Sigma}_{k}$, with rank of $r_{k}=\operatorname{rank}\left(\boldsymbol{\Sigma}_{k}\right), \boldsymbol{U}_{k} \in \mathbb{C}^{N_{B S} \times r_{k}}$ is a semi-unitary matrix consisting of the eigenvectors of $\boldsymbol{\Sigma}_{k}$ corresponding to the nonzero eigenvalues. Also, $\boldsymbol{h}_{\mathrm{NLOS}, k} \in \mathbb{C}^{r_{k} \times 1}$ denotes the random non-LOS channel for the $k^{\text {th }}$ user, whose entries are independent and identically distributed (i.i.d.) random variables and distributed according to $\mathcal{C N}(0,1)$, while $\boldsymbol{h}_{\mathrm{LOS}, k} \in \mathbb{C}^{N_{B S} \times 1}$ represents the deterministic (LOS) component, satisfying $\left\|\boldsymbol{h}_{\mathrm{LOS}, k}\right\|^{2}=N_{B S}$, where $\|\cdot\|$ denotes the Euclidean norm operator, defined as

$$
\boldsymbol{h}_{\mathrm{LOS}, k}=\left[1, e^{-j \frac{2 \pi d}{\lambda} \sin \left(\varphi_{k}\right)}, \ldots, e^{-j \frac{2 \pi d}{\lambda}\left(N_{B S}-1\right) \sin \left(\varphi_{k}\right)}\right]^{T},
$$

where $\lambda$ is the wavelength, $d$ is the inter-antenna spacing, and $\varphi_{k}$ is the $k^{\text {th }}$ user angle-of-departure (AoD), which is uniformly distributed according to $\varphi_{k} \sim \mathcal{U}\left(-\frac{\pi}{2}, \frac{\pi}{2}\right)$.

The composite transmitted signal and the received signal at the $k^{\text {th }}$ user are respectively given as

$$
\begin{gathered}
\boldsymbol{x}=\boldsymbol{F} \boldsymbol{W} \boldsymbol{P} \boldsymbol{s}=\sum_{j=1}^{U} \sqrt{P_{j}} \boldsymbol{F} \boldsymbol{w}_{j} s_{j}, \\
y_{k}=\underbrace{\sqrt{P_{k}} \boldsymbol{h}_{k}^{\dagger} \boldsymbol{F} \boldsymbol{w}_{k} s_{k}}_{\text {desired signal }}+\underbrace{\boldsymbol{h}_{k}^{\dagger} \sum_{j \neq k} \sqrt{P_{j}} \boldsymbol{F} \boldsymbol{w}_{j} s_{j}}_{\text {multiuser interference }}+\underbrace{n_{k}}_{\text {AWGN noise }},
\end{gathered}
$$

where $s \in \mathbb{C}^{U \times 1}=\left[s_{1}, s_{2}, \ldots, s_{U}\right]^{T}$ are the payload data symbols intended for the $U$ scheduled users, with entries that are i.i.d. Gaussian random variables with zero mean and unit variance, $n_{k}$ is the additive white Gaussian noise (AWGN) at the $k^{\text {th }}$ user, distributed as $n_{k} \sim \mathcal{C N}\left(0, \sigma^{2}\right)$, and $\boldsymbol{P}=\operatorname{diag}\left(\sqrt{P_{1}}, \sqrt{P_{2}}, \ldots, \sqrt{P_{U}}\right)$ is a diagonal power allocation matrix that satisfies the power constraint, i.e., the total transmit power $\sum_{j=1}^{U} P_{j}=P$. For ease of exposition, the power is equally allocated amongst users, i.e., $\boldsymbol{P}=$ $\sqrt{\frac{P}{U}} \boldsymbol{I}_{U}$, where $\boldsymbol{I}_{U}$ is the identity matrix of size $U \times U$. Hence, in this work, we normalize the analog and digital precoders such that $\left\|\boldsymbol{F} \boldsymbol{w}_{j}\right\|^{2}=1$.

Therefore, from (4), the signal-to-interference-plus-noise ratio (SINR) for the $k^{\text {th }}$ user is written as

$$
\operatorname{SINR}_{k}=\frac{\rho\left|\boldsymbol{h}_{k}^{\dagger} \boldsymbol{F} \boldsymbol{w}_{k}\right|^{2}}{1+\rho \sum_{j \neq k}\left|\boldsymbol{h}_{k}^{\dagger} \boldsymbol{F} \boldsymbol{w}_{j}\right|^{2}},
$$

where $\rho=\frac{P}{U \sigma^{2}}$ is the signal-to-noise ratio (SNR).

Our objective is to derive a closed-form analog beamformer and baseband precoder matrices such that the overall sum spectral efficiency is maximized. The achievable sum spectral efficiency of the system is expressed as

$$
\mathcal{R}_{\text {sum }}=\sum_{k=1}^{U} \mathcal{R}_{k}=\sum_{k=1}^{U} \mathbb{E}\left[\log _{2}\left(1+\operatorname{SINR}_{k}\right)\right],
$$

where $\mathbb{E}[\cdot]$ denotes the expectation operator.

Finding the optimal analog beamformer and digital precoder which maximize the achievable sum spectral efficiency in (6) is a challenging non-convex optimization problem due to the coupling between the baseband precoders $\left\{\boldsymbol{w}_{k}, k=1, \ldots, U\right\}$ in (5). As a consequence, maximum ratio transmission (MRT) and ZF schemes are two well known techniques utilized to obtain sub-optimal closedform beamformers and precoders [1], [8], [12]. Contrary, in this paper, to avoid tackling the coupled optimization problem in (6), we leverage the SLNR metric, thanks to its precoder decoupling merit, which leads to a near-optimal closed-form solution [2], [7], [10], [13].

The SLNR for the $k^{\text {th }}$ user is defined as [13]

$$
\operatorname{SLNR}_{k}=\frac{\rho\left|\boldsymbol{h}_{k}^{\dagger} \boldsymbol{F} \boldsymbol{w}_{k}\right|^{2}}{1+\rho \sum_{j \neq k}\left|\boldsymbol{h}_{j}^{\dagger} \boldsymbol{F} \boldsymbol{w}_{k}\right|^{2}} .
$$

Let $\boldsymbol{V}$ be the unconstrained analog beamformer, i.e., the amplitude and phase of its entries can be controlled, then the constrained analog beamformer $\boldsymbol{F}$ is given as [2]

$$
[\boldsymbol{F}]_{m, n}=\frac{1}{\sqrt{N_{B S}}} \exp \left(j \measuredangle[\boldsymbol{V}]_{m, n}\right),
$$

where $\measuredangle[\boldsymbol{V}]_{m, n}$ denotes the phase of the $(m, n)^{\text {th }}$ element of the matrix $\boldsymbol{V}$.

Therefore, in this paper, the optimal solution for the unconstrained analog beamformer $\boldsymbol{V}$ is investigated first, such that the constrained analog beamformer $\boldsymbol{F}$ can simply be found by (8). 


\section{FULL CSIT}

The hybrid structure makes it infeasible to acquire the entire channel of all antennas in both TDD and FDD systems due to fewer number of RF chains than BS antennas. Therefore, the full CSIT is considered here simply for benchmarking against the proposed two-stage CSIT hybrid precoder. Thus, in this section, the analog beamformer $\boldsymbol{V}$ and baseband precoder $\boldsymbol{W}$ are both designed based on the perfect knowledge of the instantaneous CSIT at the BS.

\section{A. Baseband Precoder Design}

From (7), we formulate the baseband precoder that maximizes the $\mathrm{SLNR}_{k}$ as follows

$$
\begin{array}{cc}
\boldsymbol{w}_{k}^{\star}=\underset{\boldsymbol{w}_{k}}{\arg \max } & \frac{\rho \boldsymbol{w}_{k}^{\dagger} \boldsymbol{V}^{\dagger} \boldsymbol{h}_{k} \boldsymbol{h}_{k}^{\dagger} \boldsymbol{V} \boldsymbol{w}_{k}}{\boldsymbol{w}_{k}^{\dagger}\left(\boldsymbol{V}^{\dagger} \boldsymbol{V}+\rho \sum_{j \neq k} \boldsymbol{V}^{\dagger} \boldsymbol{h}_{j} \boldsymbol{h}_{j}^{\dagger} \boldsymbol{V}\right) \boldsymbol{w}_{k}} \\
\text { s. t. } & \left\|\boldsymbol{V} \boldsymbol{w}_{k}\right\|^{2}=1
\end{array} .
$$

Proposition 1. The optimal solution to the baseband precoder in (9) is derived as

$$
\boldsymbol{w}_{k}^{\star}=\frac{\left(\boldsymbol{V}^{\dagger} \boldsymbol{V}+\rho \sum_{j \neq k} \boldsymbol{V}^{\dagger} \boldsymbol{h}_{j} \boldsymbol{h}_{j}^{\dagger} \boldsymbol{V}\right)^{-1} \boldsymbol{V}^{\dagger} \boldsymbol{h}_{k}}{\left\|\boldsymbol{V}\left(\boldsymbol{V}^{\dagger} \boldsymbol{V}+\rho \sum_{j \neq k} \boldsymbol{V}^{\dagger} \boldsymbol{h}_{j} \boldsymbol{h}_{j}^{\dagger} \boldsymbol{V}\right)^{-1} \boldsymbol{V}^{\dagger} \boldsymbol{h}_{k}\right\|} .
$$

Proof: The proof is given in Appendix A.

Substituting (10) into (7), we can obtain

$$
\mathrm{SLNR}_{k}=\rho \boldsymbol{h}_{k}^{\dagger} \boldsymbol{V}\left(\boldsymbol{V}^{\dagger} \boldsymbol{V}+\rho \sum_{j \neq k} \boldsymbol{V}^{\dagger} \boldsymbol{h}_{j} \boldsymbol{h}_{j}^{\dagger} \boldsymbol{V}\right)^{-1} \boldsymbol{V}^{\dagger} \boldsymbol{h}_{k} .
$$

\section{B. Analog Beamformer Design}

Since the analog beamformer $\boldsymbol{V}$ comprises the beamforming vectors of all users, and although several problem formulations could be investigated, for the sake of closedform solutions, we utilize the sum $\mathrm{SLNR}_{k}$ of all users to formulate the optimal analog beamformer design:

$$
\boldsymbol{V}^{\star}=\underset{\boldsymbol{V}}{\arg \max } \sum_{k=1}^{U} \mathrm{SLNR}_{k}
$$

where the $\mathrm{SLNR}_{k}$ is given in (11).

Proposition 2. A sub-optimal solution to the analog beamformer in (12) is derived as

$$
\boldsymbol{V}^{\star}=\boldsymbol{U}_{\left[1: N_{R F}\right]}\left[\sum_{k=1}^{U}\left(\boldsymbol{I}_{N_{B S}}+\rho \sum_{j=1}^{U} \boldsymbol{h}_{j} \boldsymbol{h}_{j}^{\dagger}\right)^{-1} \boldsymbol{h}_{k} \boldsymbol{h}_{k}^{\dagger}\right]
$$

where $\boldsymbol{U}_{\left[1: N_{R F}\right]}=\boldsymbol{U}\left(:, 1: N_{R F}\right)$ is a $N_{B S} \times$ $N_{R F}$ matrix whose columns are the $N_{R F}$ leading principal components (eigenvectors) corresponding to the $N_{R F}$ strongest eigenvalues of the matrix $\sum_{k=1}^{U}\left(\boldsymbol{I}_{N_{B S}}+\rho \sum_{j=1}^{U} \boldsymbol{h}_{j} \boldsymbol{h}_{j}^{\dagger}\right)^{-1} \boldsymbol{h}_{k} \boldsymbol{h}_{k}^{\dagger}$.

Proof: The proof is given in Appendix B.

\section{STATISTICAL CSIT}

Statistical CSIT is preferable in FDD massive MIMO and leveraged here as it varies over a longer timescale when compared to the instantaneous channel variations, hence, substantially reducing the required training sequences and feedback overhead. However, a residual amount of multiuser interference remains due to the impact of unknown instantaneous multipath components, resulting in a performance saturation particularly at high SNR. Therefore, in this section, the analog beamformer $\boldsymbol{V}$ and digital precoder $\boldsymbol{W}$ are designed based only on the long-term first and second order channel statistics information via the maximization of a lower-bound on the average SLNR.

A lower-bound on the average SLNR for the $k^{\text {th }}$ user is derived as

$$
\mathbb{E}\left[\mathrm{SLNR}_{k}\right] \geq \frac{\rho \boldsymbol{w}_{k}^{\dagger} \boldsymbol{V}^{\dagger} \boldsymbol{R}_{k} \boldsymbol{V} \boldsymbol{w}_{k}}{\boldsymbol{w}_{k}^{\dagger}\left(\boldsymbol{V}^{\dagger} \boldsymbol{V}+\rho \sum_{j \neq k} \boldsymbol{V}^{\dagger} \boldsymbol{R}_{j} \boldsymbol{V}\right) \boldsymbol{w}_{k}},
$$

where $\boldsymbol{R}_{k}=\frac{K_{k}}{K_{k}+1} \boldsymbol{h}_{\mathrm{LOS}, k} \boldsymbol{h}_{\mathrm{LOS}, k}^{\dagger}+\frac{1}{K_{k}+1} \boldsymbol{\Sigma}_{k}$.

The derivation of a lower-bound on $\mathbb{E}\left[\mathrm{SLNR}_{k}\right]$ follows similar steps to [7, Eq. (10)]

\section{A. Baseband Precoder Design}

From (14), the baseband precoder design problem is formulated as

$$
\begin{array}{cc}
\boldsymbol{w}_{k}^{\star}=\underset{\boldsymbol{w}_{k}}{\arg \max } & \frac{\rho \boldsymbol{w}_{k}^{\dagger} \boldsymbol{V}^{\dagger} \boldsymbol{R}_{k} \boldsymbol{V} \boldsymbol{w}_{k}}{\boldsymbol{w}_{k}^{\dagger}\left(\boldsymbol{V}^{\dagger} \boldsymbol{V}+\rho \sum_{j \neq k} \boldsymbol{V}^{\dagger} \boldsymbol{R}_{j} \boldsymbol{V}\right) \boldsymbol{w}_{k}} \\
\text { s.t. } & \left\|\boldsymbol{V} \boldsymbol{w}_{k}\right\|^{2}=1
\end{array} .
$$

Proposition 3. The optimal baseband precoder that maximizes (15) is derived as

$$
\boldsymbol{w}_{k}^{\star}=\frac{\boldsymbol{u}_{\max }\left[\left(\boldsymbol{V}^{\dagger} \boldsymbol{V}+\rho \sum_{j \neq k} \boldsymbol{V}^{\dagger} \boldsymbol{R}_{j} \boldsymbol{V}\right)^{-1} \boldsymbol{V}^{\dagger} \boldsymbol{R}_{k} \boldsymbol{V}\right]}{\left\|\boldsymbol{V} \boldsymbol{u}_{\max }\left[\left(\boldsymbol{V}^{\dagger} \boldsymbol{V}+\rho \sum_{j \neq k} \boldsymbol{V}^{\dagger} \boldsymbol{R}_{j} \boldsymbol{V}\right)^{-1} \boldsymbol{V}^{\dagger} \boldsymbol{R}_{k} \boldsymbol{V}\right]\right\|_{(16)}},
$$

where $\boldsymbol{u}_{\max }[\boldsymbol{X}]$ is the dominant eigenvector of the matrix $\boldsymbol{X}$.

Proof: The proof follows similar steps to the derivation of Proposition 1.

Substituting (16) into (14), reduces to

$\mathbb{E}\left[\mathrm{SLNR}_{k}\right] \geq \rho \lambda_{\max }\left[\left(\boldsymbol{V}^{\dagger} \boldsymbol{V}+\rho \sum_{j \neq k} \boldsymbol{V}^{\dagger} \boldsymbol{R}_{j} \boldsymbol{V}\right)^{-1} \boldsymbol{V}^{\dagger} \boldsymbol{R}_{k} \boldsymbol{V}\right]$,

where $\lambda_{\max }[\boldsymbol{X}]$ is the strongest eigenvalue of the matrix $\boldsymbol{X}$.

\section{B. Analog Beamformer Design}

The optimization problem for the analog RF beamformer $\boldsymbol{V}^{\star}$ is formulated as

$$
\boldsymbol{V}^{\star}=\underset{\boldsymbol{V}}{\arg \max } \sum_{k=1}^{U} \mathbb{E}\left[\mathrm{SLNR}_{k}\right],
$$

where the lower-bound on $\mathbb{E}\left[\mathrm{SLNR}_{k}\right]$ is given in (17). 
Proposition 4. A sub-optimal solution to the analog beamformer problem in (18) is derived as

$$
\boldsymbol{V}^{\star}=\boldsymbol{U}_{\left[1: N_{R F}\right]}\left[\sum_{k=1}^{U}\left(\boldsymbol{I}_{N_{B S}}+\rho \sum_{j=1}^{U} \boldsymbol{R}_{j}\right)^{-1} \boldsymbol{R}_{k}\right] .
$$

Proof: The proof is given in Appendix C.

\section{Two-STAGE CSIT}

The estimation and feedback overhead of the channel statistics information at the transmitter can be easily and accurately acquired through long-term downlink training sequences and low-rate uplink feedback. ${ }^{1}$ As was previously mentioned, relying exclusively on statistical CSIT creates a performance bottleneck, due to the remaining multiuser interference; this, in turn, creates a saturation of the spectral efficiency at high SNRs. Therefore, to alleviate this issue, a two-stage precoding design technique is developed here, where the analog beamformer stage is designed based on the knowledge of statistical CSIT to steer the beams towards the dominant eigendirections of the desired users while eliminating the impact of multiuser interference in the statistical sense, while the baseband precoder stage eliminates the multiuser interference utilizing only the instantaneous knowledge of a reduced dimensionality effective channel.

In particular, in the two-stage CSIT case, the analog beamformer $\boldsymbol{V}$ is designed exploiting only $\boldsymbol{R}_{k} \forall k \in$ $\{1,2, \ldots, U\}$ and $\rho$, while the digital precoder design $\boldsymbol{W}$ utilizes the reduced dimensionality instantaneous CSIT of the effective channel $\boldsymbol{H}_{\text {eff }}=\boldsymbol{V}^{\dagger} \boldsymbol{H}$, where $\boldsymbol{H}=$ $\left[\boldsymbol{h}_{1}, \boldsymbol{h}_{2}, \ldots, \boldsymbol{h}_{U}\right]$. Hence, rather than acquiring $N_{B S} \times U$ instantaneous CSIT elements for $\boldsymbol{H}$, the BS obtains only $N_{R F} \times U$ instantaneous CSIT elements for $\boldsymbol{H}_{\text {eff }}$ instead. This is a huge overhead reduction for the estimation and feedback of the instantaneous CSIT as the downlink training and uplink feedback overhead scales down to the number of RF chains (i.e., close to the active users) rather than the massive number of BS antennas.

\section{A. Baseband Precoder Design}

The baseband precoder is designed based on the knowledge of the reduced dimensionality effective channel $\boldsymbol{H}_{\text {eff }}=$ $\boldsymbol{V}^{\dagger} \boldsymbol{H}$. As such, the two-stage baseband precoder problem formulation and derivations follow similar steps to that of the full CSI case in (9) and (10).

Proposition 5. The optimal two-stage baseband precoder is given as

$$
\boldsymbol{w}_{k}^{\star}=\frac{\left(\boldsymbol{V}^{\dagger} \boldsymbol{V}+\rho \sum_{j \neq k} \boldsymbol{V}^{\dagger} \boldsymbol{h}_{j} \boldsymbol{h}_{j}^{\dagger} \boldsymbol{V}\right)^{-1} \boldsymbol{V}^{\dagger} \boldsymbol{h}_{k}}{\left\|\boldsymbol{V}\left(\boldsymbol{V}^{\dagger} \boldsymbol{V}+\rho \sum_{j \neq k} \boldsymbol{V}^{\dagger} \boldsymbol{h}_{j} \boldsymbol{h}_{j}^{\dagger} \boldsymbol{V}\right)^{-1} \boldsymbol{V}^{\dagger} \boldsymbol{h}_{k}\right\|}
$$

\footnotetext{
${ }^{1}$ Statistical channel information is reciprocal for both FDD and TDD systems [14], [15], potentially eliminating the need for downlink channel statistics estimation and feedback in FDD systems, which results in significant training and feedback overhead reduction.
}

Proof: This is similar to the derived optimal baseband precoder in (10).

Note that here in contrast to (10), the analog beamformer $\boldsymbol{V}$ in (20) relies on statistical CSIT. Therefore, substituting (20) into (7) results in (11), whose average is lower-bounded as

$$
\begin{aligned}
& \mathbb{E}\left[\mathrm{SLNR}_{k}\right] \\
= & \mathbb{E}\left[\rho \boldsymbol{h}_{k}^{\dagger} \boldsymbol{V}\left(\boldsymbol{V}^{\dagger} \boldsymbol{V}+\rho \sum_{j \neq k} \boldsymbol{V}^{\dagger} \boldsymbol{h}_{j} \boldsymbol{h}_{j}^{\dagger} \boldsymbol{V}\right)^{-1} \boldsymbol{V}^{\dagger} \boldsymbol{h}_{k}\right] \\
= & \rho \mathbb{E}\left[\operatorname{Tr}\left(\left(\boldsymbol{V}^{\dagger} \boldsymbol{V}+\rho \sum_{j \neq k} \boldsymbol{V}^{\dagger} \boldsymbol{h}_{j} \boldsymbol{h}_{j}^{\dagger} \boldsymbol{V}\right)^{-1} \boldsymbol{V}^{\dagger} \boldsymbol{h}_{k} \boldsymbol{h}_{k}^{\dagger} \boldsymbol{V}\right)\right] \\
\geq & \rho \operatorname{Tr}\left(\left(\boldsymbol{V}^{\dagger} \boldsymbol{V}+\rho \sum_{j \neq k} \boldsymbol{V}^{\dagger} \boldsymbol{R}_{j} \boldsymbol{V}\right)^{-1} \boldsymbol{V}^{\dagger} \boldsymbol{R}_{k} \boldsymbol{V}\right),
\end{aligned}
$$

where $\operatorname{Tr}(\boldsymbol{X})$ denotes the trace of the matrix $\boldsymbol{X}$, the lowerbound in (21) is obtained by utilizing the Jensen's inequality through the convexity of the function.

\section{B. Analog Beamformer Design}

The design of the analog beamformer $\boldsymbol{V}$ relies only on the long-term channel statistics information, hence, similar to (18), the design metric for the analog beamformer is given as

$$
\boldsymbol{V}^{\star}=\underset{\boldsymbol{V}}{\arg \max } \sum_{k=1}^{U} \mathbb{E}\left[\mathrm{SLNR}_{k}\right] .
$$

Proposition 6. A sub-optimal solution to the analog beamformer in (22) is derived as

$$
\boldsymbol{V}^{\star}=\boldsymbol{U}_{\left[1: N_{R F}\right]}\left[\sum_{k=1}^{U}\left(\boldsymbol{I}_{N_{B S}}+\rho \sum_{j=1}^{U} \boldsymbol{R}_{j}\right)^{-1} \boldsymbol{R}_{k}\right] \text {. }
$$

Proof: The proof follows similar steps to the derivations of (19).

\section{NumericAl RESUlts}

In this section, a set of numerical results are provided to evaluate the performance of the proposed analog beamformers and baseband precoders. The one-ring local scattering correlation model is used here, where the $(m, n)^{\text {th }}$ entry of $\Sigma_{k}$ is given as [16]

$$
\left[\boldsymbol{\Sigma}_{k}\right]_{m, n}=\frac{1}{2 \Delta_{k}} \int_{\phi_{k}-\Delta_{k}}^{\phi_{k}+\Delta_{k}} e^{-j \frac{2 \pi d}{\lambda}(n-m) \sin \left(\theta_{k}\right)} d \theta_{k},
$$

where $\Delta_{k}$ denotes the $k^{\text {th }}$ user azimuth angular spread, $\phi_{k}$ is the $k^{\text {th }}$ user central azimuth angle, and $\theta_{k}$ is the $k^{\text {th }}$ user actual AoD, distributed according to $\theta_{k} \sim$ $\mathcal{U}\left(\phi_{k}-\Delta_{k}, \phi_{k}+\Delta_{k}\right)$. In our simulations, we set $\phi_{k} \sim$ $\mathcal{U}\left(-\frac{\pi}{2}, \frac{\pi}{2}\right)$. 


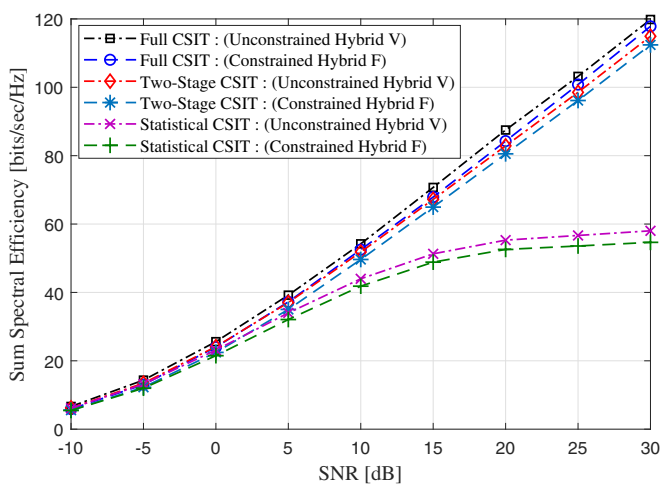

Figure 1. The sum spectral efficiency versus the SNR for the unconstrained analog beamformer $\boldsymbol{V}$ and constrained analog beamformer $\boldsymbol{F}$, with Ricean $\mathrm{K}$-factor $K_{k}=10 \mathrm{~dB} \forall k, \Delta_{k}=15^{\circ} \forall k, N_{B S}=64$ and $U=N_{R F}=$ 10 users.

In Fig. 1, the achievable sum spectral efficiency of the proposed analog beamformers and digital precoders are shown. It is to be emphasized that the constrained analog beamformer $\boldsymbol{F}$ is derived from the unconstrained analog beamformer $\boldsymbol{V}$ based on (8). It is clearly seen that the achievable sum spectral efficiency of the proposed two-stage CSIT beamformer in (6) with the analog beamformer in (23) and baseband precoder in (20) is very close to its full CSIT counterpart with analog beamformer in (13) and baseband precoder in (10), while obviously seen that the sum spectral efficiency of statistical CSIT with the analog beamformer in (19) and baseband precoder in (16) saturates at high SNR. The performance gap between the unconstrained hybrid precoding $\boldsymbol{V}$ and constrained hybrid precoding $\boldsymbol{F}$ is shown to be negligible.

The proposed two-stage CSIT performance comes with a remarkably less number of downlink training sequences and uplink feedback overhead. For instance, traditionally, the full CSIT case acquires $N_{B S} \times U$ instantaneous CSIT elements for $\boldsymbol{H}$ at the BS, which requires $N_{B S} \times U$ downlink training sequences and a comparable uplink feedback overhead. Utilizing the low rank structure of massive MIMO systems, the full CSIT case acquires only $\sum_{k=1}^{U} r_{k}$ instantaneous CSIT elements for $\boldsymbol{H}$ at the BS. However, the twostage CSIT scenario acquires only $N_{R F} \times U \ll \sum_{k=1}^{U} r_{k}$ instantaneous CSIT elements for $\boldsymbol{H}_{\text {eff }}=\boldsymbol{V}^{\dagger} \boldsymbol{H}$ at the BS (Note that $\boldsymbol{V}$ here relies on statistical CSIT), which simply requires $N_{R F} \times U$ downlink training sequences. This is an exceptional reduction in the number of downlink training sequences and uplink feedback overhead. Meanwhile, the statistical CSIT scenario has the least overhead burden as the channel mean and covariance statistics remain fixed over multiple channel coherence intervals.

Fig. 2 presents the sum spectral efficiency versus the Ricean K-factor for the unconstrained analog beamformer $\boldsymbol{V}$. In contrast to the full CSIT scenario, the Ricean K-factor has a higher impact on the statistical and two-stage CSIT scenarios, where a significant performance improvement is noticed as a result of higher Ricean $\mathrm{K}$-factor. This is expected as the statistical CSIT case relies solely on the first

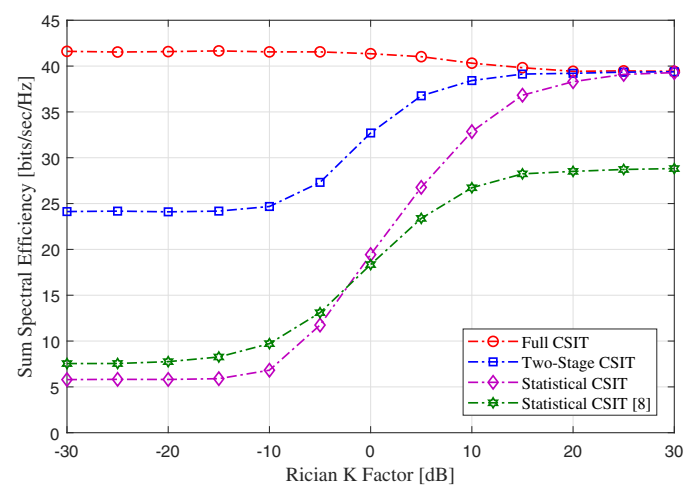

Figure 2. The sum spectral efficiency versus the Ricean K-factor for the unconstrained analog beamformer $\boldsymbol{V}$, with $\Delta_{k}=10^{\circ} \forall k, \rho=10 \mathrm{~dB}$, $N_{B S}=32$ and $U=N_{R F}=10$ users.

and second order channel statistics information, whereas in the two-stage CSIT scenario, only the analog RF beamforming stage depends on channel statistics information. For benchmarking with the literature, the beamformer derived in $[8$, Theorem 1] is considered.

\section{CONCLUSIONS}

A two-stage hybrid precoding technique that leverages only the long-term channel statistics information to derive the analog beamformer, whereas utilized perfect CSIT of the reduced dimensionality effective channel for the baseband precoder design was proposed. An optimal closed-form baseband precoder was first derived as a function of the analog beamformer by exploiting the instantaneous SLNR metric. Then, based on the derived baseband precoder, a sub-optimal closed-form analog beamformer was presented utilizing the average SLNR criterion. It was clearly shown that the sum spectral efficiency of the fully digital precoding structure performs only marginally better than that of the hybrid analog and digital precoding structure. Furthermore, the sum spectral efficiency performance of the proposed two-stage CSIT beamforming is very close to that of the full CSIT beamforming with the merit of outstanding reduction in CSIT acquisition and feedback overhead.

\section{APPENDIX A}

PROOF FOR PROPOSITION 1

An equivalent desired formulation for the unconstrained problem in (9) can be re-written as

$$
\begin{array}{cc}
\boldsymbol{w}_{k}^{\star}=\underset{\boldsymbol{w}_{k}}{\arg \max } & \boldsymbol{w}_{k}^{\dagger} \boldsymbol{V}^{\dagger} \boldsymbol{h}_{k} \boldsymbol{h}_{k}^{\dagger} \boldsymbol{V} \boldsymbol{w}_{k} \\
\text { s. t. } & \boldsymbol{w}_{k}^{\dagger}\left(\boldsymbol{V}^{\dagger} \boldsymbol{V}+\rho \sum_{j \neq k} \boldsymbol{V}^{\dagger} \boldsymbol{h}_{j} \boldsymbol{h}_{j}^{\dagger} \boldsymbol{V}\right) \boldsymbol{w}_{k}=c
\end{array}
$$

where $c$ is constant.

This problem can be easily solved using the Lagrange multiplier, as follows

$$
\begin{aligned}
L\left(\boldsymbol{w}_{k}\right) & =\boldsymbol{w}_{k}^{\dagger} \boldsymbol{V}^{\dagger} \boldsymbol{h}_{k} \boldsymbol{h}_{k}^{\dagger} \boldsymbol{V} \boldsymbol{w}_{k}- \\
& \kappa\left(\boldsymbol{w}_{k}^{\dagger}\left(\boldsymbol{V}^{\dagger} \boldsymbol{V}+\rho \sum_{j \neq k} \boldsymbol{V}^{\dagger} \boldsymbol{h}_{j} \boldsymbol{h}_{j}^{\dagger} \boldsymbol{V}\right) \boldsymbol{w}_{k}-c\right)
\end{aligned}
$$


where $\kappa$ is the Lagrange multiplier.

Therefore, once we maximize with respect to $\boldsymbol{w}_{k}$, we get

$$
\boldsymbol{V}^{\dagger} \boldsymbol{h}_{k} \boldsymbol{h}_{k}^{\dagger} \boldsymbol{V} \boldsymbol{w}_{k}=\kappa\left(\boldsymbol{V}^{\dagger} \boldsymbol{V}+\rho \sum_{j \neq k} \boldsymbol{V}^{\dagger} \boldsymbol{h}_{j} \boldsymbol{h}_{j}^{\dagger} \boldsymbol{V}\right) \boldsymbol{w}_{k} .
$$

This is a generalized eigenvalue problem, i.e., $\boldsymbol{w}_{k}^{\star}$ is the generalized eigenvector corresponding to the largest generalized eigenvalue $\lambda_{\max }$ of $\left\{\boldsymbol{V}^{\dagger} \boldsymbol{h}_{k} \boldsymbol{h}_{k}^{\dagger} \boldsymbol{V}, \boldsymbol{V}^{\dagger} \boldsymbol{V}+\rho \sum_{j \neq k} \boldsymbol{V}^{\dagger} \boldsymbol{h}_{j} \boldsymbol{h}_{j}^{\dagger} \boldsymbol{V}\right\}$ Since $\left(\boldsymbol{V}^{\dagger} \boldsymbol{V}+\rho \sum_{j \neq k} \boldsymbol{V}^{\dagger} \boldsymbol{h}_{j} \boldsymbol{h}_{j}^{\dagger} \boldsymbol{V}\right)$ is invertible, the generalized eigenvalue problem (27) reduces to the standard eigenvalue problem

$$
\left(\boldsymbol{V}^{\dagger} \boldsymbol{V}+\rho \sum_{j \neq k} \boldsymbol{V}^{\dagger} \boldsymbol{h}_{j} \boldsymbol{h}_{j}^{\dagger} \boldsymbol{V}\right)^{-1} \boldsymbol{V}^{\dagger} \boldsymbol{h}_{k} \boldsymbol{h}_{k}^{\dagger} \boldsymbol{V} \boldsymbol{w}_{k}=\kappa \boldsymbol{w}_{k} .
$$

Therefore, the optimal baseband precoder is the normalized dominant eigenvector of the matrix $\left(\boldsymbol{V}^{\dagger} \boldsymbol{V}+\rho \sum_{j \neq k} \boldsymbol{V}^{\dagger} \boldsymbol{h}_{j} \boldsymbol{h}_{j}^{\dagger} \boldsymbol{V}\right)^{-1} \boldsymbol{V}^{\dagger} \boldsymbol{h}_{k} \boldsymbol{h}_{k}^{\dagger} \boldsymbol{V}$ Hence, once taking the normalization into account, (28) reduces to (10), and this concludes the proof.

\section{APPENDIX B}

\section{PROOF FOR PROPOSITION 2}

Utilizing the identity $\boldsymbol{x}^{\dagger} \boldsymbol{A} \boldsymbol{x}=\operatorname{Tr}\left(\boldsymbol{A} \boldsymbol{x} \boldsymbol{x}^{\dagger}\right)$, the sum $\mathrm{SLNR}_{k}$ is re-written as

$$
\begin{aligned}
& \sum_{k=1}^{U} \operatorname{SLNR}_{k}=\sum_{k=1}^{U} \rho \boldsymbol{h}_{k}^{\dagger} \boldsymbol{V}\left(\boldsymbol{V}^{\dagger} \boldsymbol{V}+\rho \sum_{j \neq k} \boldsymbol{V}^{\dagger} \boldsymbol{h}_{j} \boldsymbol{h}_{j}^{\dagger} \boldsymbol{V}\right)^{-1} \boldsymbol{V}^{\dagger} \boldsymbol{h}_{k} \\
& \geq \rho \operatorname{Tr}\left(\sum_{k=1}^{U}\left(\boldsymbol{V}^{\dagger} \boldsymbol{V}+\rho \sum_{j=1}^{U} \boldsymbol{V}^{\dagger} \boldsymbol{h}_{j} \boldsymbol{h}_{j}^{\dagger} \boldsymbol{V}\right)^{-1} \boldsymbol{V}^{\dagger} \boldsymbol{h}_{k} \boldsymbol{h}_{k}^{\dagger} \boldsymbol{V}\right)
\end{aligned}
$$

where the inequality in (29) comes from the rank-1 perturbation lemma. Hence, a sub-optimal solution to the analog beamformer $\boldsymbol{V}$ is given as

$$
\begin{aligned}
& \boldsymbol{V}^{\star}= \\
& \underset{\boldsymbol{V}}{\arg \max } \operatorname{Tr}\left(\sum_{k=1}^{U}\left(\boldsymbol{V}^{\dagger} \boldsymbol{V}+\rho \sum_{j=1}^{U} \boldsymbol{V}^{\dagger} \boldsymbol{h}_{j} \boldsymbol{h}_{j}^{\dagger} \boldsymbol{V}\right)^{-1} \boldsymbol{V}^{\dagger} \boldsymbol{h}_{k} \boldsymbol{h}_{k}^{\dagger} \boldsymbol{V}\right) .
\end{aligned}
$$

With the aid of (27)-(28), (30) reduces to [17, Eq. (7)]

$$
\begin{aligned}
& \boldsymbol{V}^{\star}= \\
& \underset{\boldsymbol{V}^{\dagger} \boldsymbol{V}=\boldsymbol{I}}{\arg \max } \operatorname{Tr}\left(\boldsymbol{V}^{\dagger} \sum_{k=1}^{U}\left(\boldsymbol{I}_{N_{B S}}+\rho \sum_{j=1}^{U} \boldsymbol{h}_{j} \boldsymbol{h}_{j}^{\dagger}\right)^{-1} \boldsymbol{h}_{k} \boldsymbol{h}_{k}^{\dagger} \boldsymbol{V}\right) .
\end{aligned}
$$

The optimal solution to (31) is the $N_{B S} \times N_{R F}$ matrix containing the $N_{R F}$ leading eigenvectors associated with the $N_{R F}$ strongest eigenvalues of the matrix $\sum_{k=1}^{U}\left(\boldsymbol{I}_{N_{B S}}+\rho \sum_{j \neq k} \boldsymbol{h}_{j} \boldsymbol{h}_{j}^{\dagger}\right)^{-1} \boldsymbol{h}_{k} \boldsymbol{h}_{k}^{\dagger}$ [17].

\section{APPENDIX C}

\section{PROOF FOR PROPOSITION 4}

We first recall the identity

$$
\sum_{k=1}^{U} \lambda_{\max }\left(\boldsymbol{X}_{k}\right) \leq \operatorname{Tr}\left(\sum_{k=1}^{U} \boldsymbol{X}_{k}\right)
$$

From (17)-(18), a sub-optimal formulation for the analog beamformer $\boldsymbol{V}^{\star}$ is defined as

$$
\begin{gathered}
\boldsymbol{V}^{\star}= \\
\underset{\boldsymbol{V}}{\arg \max } \sum_{k=1}^{U} \operatorname{Tr}\left(\left(\boldsymbol{V}^{\dagger} \boldsymbol{V}+\rho \sum_{j \neq k} \boldsymbol{V}^{\dagger} \boldsymbol{R}_{j} \boldsymbol{V}\right)^{-1} \boldsymbol{V}^{\dagger} \boldsymbol{R}_{k} \boldsymbol{V}\right) .
\end{gathered}
$$

Consequently, by following similar steps with the derivation of (13), we arrive at (19), and this concludes the proof.

\section{REFERENCES}

[1] A. Alkhateeb, G. Leus, and R. W. Heath, Jr., "Limited feedback hybrid precoding for multi-user millimeter wave systems," IEEE Trans. Wireless Commun., vol. 14, no. 11, pp. 6481-6494, Nov. 2015.

[2] S. Park, J. Park, A. Yazdan, and R. W. Heath, Jr., "Exploiting spatial channel covariance for hybrid precoding in massive MIMO systems," IEEE Trans. Signal Process., vol. 65, no. 14, pp. 3818-3832, Jul. 2017.

[3] A. F. Molisch, V. V. Ratnam, S. Han, Z. Li, S. L. H. Nguyen, L. Li, and K. Haneda, "Hybrid beamforming for massive MIMO: A survey," IEEE Commun. Mag., vol. 55, no. 9, pp. 134-141, Sep. 2017.

[4] A. Liu and V. K. N. Lau, "Phase only RF precoding for massive MIMO systems with limited RF chains," IEEE Trans. Signal Process., vol. 62, no. 17, pp. 4505-4515, Sep. 2014.

[5] A. Adhikary, J. Nam, J. Ahn, and G. Caire, "Joint spatial division and multiplexing - The large-scale array regime," IEEE Trans. Inf. Theory, vol. 59, no. 10, pp. 6441-6463, Oct. 2013.

[6] X. Rao and V. K. N. Lau, "Distributed compressive CSIT estimation and feedback for FDD multi-user massive MIMO systems," IEEE Trans. Signal Process., vol. 62, no. 12, pp. 3261-3271, Jun. 2014.

[7] M. Dai and B. Clerckx, "Multiuser millimeter wave beamforming strategies with quantized and statistical CSIT," IEEE Trans. Wireless Commun., vol. 16, no. 11, pp. 7025-7038, Nov. 2017.

[8] S. Jin, W. Tan, M. Matthaiou, J. Wang, and K. K. Wong, "Statistical eigenmode transmission for the MU-MIMO downlink in Rician fading," IEEE Trans. Wireless Commun., vol. 14, no. 12, pp. 66506663, Dec. 2015.

[9] I. Boukhedimi, A. Kammoun, and M. S. Alouini, "LMMSE receivers in uplink massive MIMO systems with correlated Rician fading," IEEE Trans. Commun., vol. 67, no. 1, pp. 230-243, Jan. 2019.

[10] D. Kim, G. Lee, and Y. Sung, "Two-stage beamformer design for massive MIMO downlink by trace quotient formulation," IEEE Trans. Commun., vol. 63, no. 6, pp. 2200-2211, Jun. 2015.

[11] A. Almradi, M. Matthaiou, and V. Fusco, "Two-stage limited feedback hybrid precoding in multiuser MIMO systems," in Proc. IEEE ICC 2020, To Appear, Accepted.

[12] F. Sohrabi and W. Yu, "Hybrid digital and analog beamforming design for large-scale antenna arrays," IEEE J. Sel. Topics Signal Process., vol. 10, no. 3, pp. 501-513, Apr. 2016.

[13] M. Sadek, A. Tarighat, and A. H. Sayed, "A leakage-based precoding scheme for downlink multi-user MIMO channels," IEEE Trans. Wireless Commun., vol. 6, no. 5, pp. 1711-1721, May 2007.

[14] H. Xie, F. Gao, S. Jin, J. Fang, and Y. Liang, "Channel estimation for TDD/FDD massive MIMO systems with channel covariance computing," IEEE Trans. Wireless Commun., vol. 17, no. 6, pp. 42064218, June 2018.

[15] M. Barzegar Khalilsarai, S. Haghighatshoar, X. Yi, and G. Caire, "FDD massive MIMO via UL/DL channel covariance extrapolation and active channel sparsification," IEEE Trans. Wireless Commun., vol. 18, no. 1, pp. 121-135, Jan. 2019.

[16] A. Adhikary, E. Al Safadi, M. K. Samimi, R. Wang, G. Caire, T. S. Rappaport, and A. F. Molisch, "Joint spatial division and multiplexing for mm-wave channels," IEEE J. Sel. Areas Commun., vol. 32, no. 6, pp. 1239-1255, June 2014.

[17] H. Wang, S. Yan, D. Xu, X. Tang, and T. Huang, "Trace ratio vs. ratio trace for dimensionality reduction," in Proc. IEEE CVPR, June 2007, pp. 1-8. 